\title{
NMR and molecular dynamics studies combined to anti-cancer nano drugs and DNA/RNA interactions in gum cancer cells and their modulations with resistance mutations
}

\author{
Alireza Heidari ${ }^{1,2 *}$ and Ricardo Gobato ${ }^{3}$ \\ ${ }^{1}$ Faculty of Chemistry, California South University, 14731 Comet St. Irvine, CA 92604, USA \\ ${ }^{2}$ American International Standards Institute, Irvine, CA 3800, USA \\ ${ }^{3}$ Green Land Landscaping and Gardening, Seedling Growth Laboratory, 86130-000, Parana, Brazil
}

\begin{abstract}
In the current research, NMR and molecular dynamics studies combined to anti-cancer Nano drugs and DNA/RNA interactions in gum cancer cells and their modulations with resistance mutations are considered. The early diagnosis of cancer is very important for patient treatment and recovery. If cancers can be confirmed and classified at an early stage, the patient survival rate can be greatly improved. Current clinical cancer diagnostic techniques can be divided into two catalogs: tissue imaging and NMR and molecular dynamics studies combined to anti-cancer Nano drugs and DNA/RNA interactions in gum cancer cells and their modulations with resistance mutations.
\end{abstract}

\section{Introduction}

Biomarkers can be used for cancer identification in most cases. Some biomarkers exhibit resonance with $\mathrm{THz}$ waves, so NMR and molecular dynamics studies combined to anti-cancer Nano drugs and DNA/RNA interactions in gum cancer cells and their modulations with resistance mutations can be a tool for biomarker identification in cancer tissue. However, the low concentrations of biomarkers and the presence of various outer substances in the cancer tissue make it difficult to identify biomarkers in real tissues. Therefore, current NMR and molecular dynamics studies combined to anti-cancer Nano drugs and DNA/RNA interactions in gum cancer cells and their modulations with resistance mutations technologies can only identify biomarkers in engineered mixtures with no more than ten substances. NMR and molecular dynamics studies combined to anti-cancer Nano drugs and DNA/RNA interactions in gum cancer cells and their modulations with resistance mutations is an inelastic scattering process in which photons incident on a sample transfer energy to or from molecular vibrational modes. It is a coherent two-photon process in which a molecule simultaneously absorbs an incident photon and emits a NMR and molecular dynamics studies combined to anti-cancer Nano drugs and DNA/RNA interactions in gum cancer cells and their modulations with resistance mutations, accompanied by its transition from one energy level to another, giving rise to a frequency (i.e., energy) shift of the emitted photon. Because the energy levels are unique for every molecule, NMR and molecular dynamics studies combined to anti-cancer Nano drugs and DNA/RNA interactions in gum cancer cells and their modulations with resistance mutations are chemicalspecific. Individual bands in the NMR and molecular dynamics studies combined to anti-cancer Nano drugs and DNA/RNA interactions in gum cancer cells and their modulations with resistance mutations are characteristic of specific molecular motions. NMR and molecular dynamics studies combined to anti-cancer Nano drugs and DNA/RNA interactions in gum cancer cells and their modulations with resistance mutations is particularly amenable to in vivo measurements, because the powers and excitation wavelengths that are used do not affect the tissue and the penetration depth is relatively large [1-10].

\section{Results and discussion}

NMR and molecular dynamics studies combined to anti-cancer Nano drugs and DNA/RNA interactions in gum cancer cells and their modulations with resistance mutations presented here have demonstrated the feasibility of using $\mathrm{THz}$ technologies in cancer diagnosis. However, additional development is needed before these technologies can be practically applied for the early diagnosis of cancer. Future developments of $\mathrm{THz}$ technologies for cancer diagnosis include combining $\mathrm{THz}$ imaging with $\mathrm{THz}$ NMR and molecular dynamics studies combined to anti-cancer Nano drugs and DNA/ RNA interactions in gum cancer cells and their modulations with resistance mutations fingerprinting of biomarkers to realize qualitative identification and quantitative analysis simultaneously. To achieve this

*Correspondence to: Alireza Heidari, Faculty of Chemistry, California South University, 14731 Comet St. Irvine, CA 92604; American International Standards Institute, Irvine, CA 3800, USA, E-mail: Scholar.Researcher.Scientist@gmail. com; Alireza.Heidari@calsu.us; Central@aisi-usa.org

Key words: NMR, molecular dynamics, anti-cancer nano drugs, DNA/RNA interactions, gum cancer cells, modulations, resistance mutations

Received: September 28, 2020; Accepted: October 12, 2020, Published: October 16,2020 

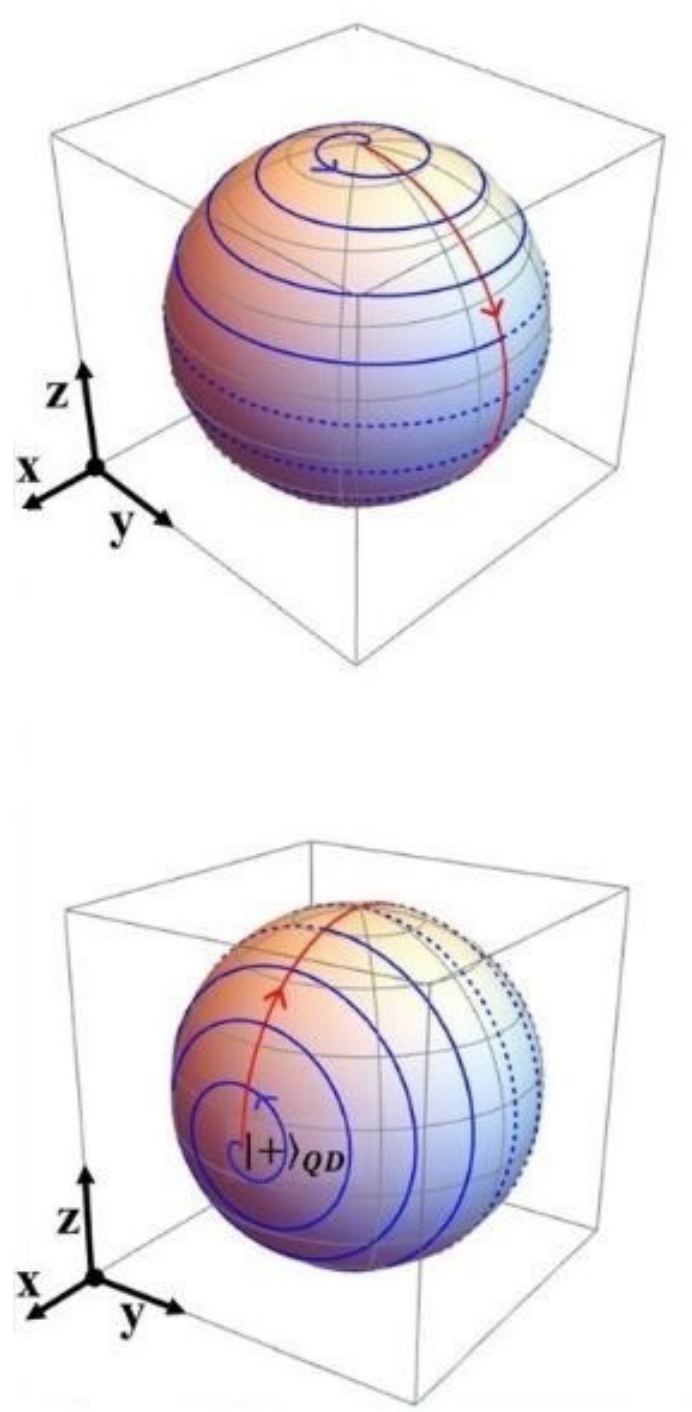

Figure 1. NMR and molecular dynamics studies combined to anti-cancer Nano drugs and DNA/RNA interactions in gum cancer cells and their modulations with resistance mutations

objective, further improvements in $\mathrm{THz}$ systems and auxiliary methods are needed (Figure 1).

\section{Conclusion}

NMR and molecular dynamics studies combined to anti-cancer Nano drugs and DNA/RNA interactions in gum cancer cells and their modulations with resistance mutations have been developed to address these issues. These methods included the use of contrast agents, optical cleaning agents, antibodies, and metamaterials to enhance the NMR and molecular dynamics studies combined to anti-cancer Nano drugs and DNA/RNA interactions in gum cancer cells and their modulations with resistance mutations. However, sample contamination by additional substances is unavoidable. Antibodies have been combined with metamaterial biosensors to achieve highly sensitive biomarker detection. However, the process of developing antibodies for biomarkers is complicated, and antibodies may not be available for some cancer biomarkers.

\section{Acknowledgments}

This study was supported by the Cancer Research Institute (CRI) Project of Scientific Instrument and Equipment Development, the National Natural Science Foundation of the United Sates, the International Joint BioSpectroscopy Core Research Laboratory Program supported by the California South University (CSU), and the Key project supported by the American International Standards Institute (AISI), Irvine, California, USA.

\section{References}

1. Heidari A, Brown C (2015) Study of composition and morphology of cadmium oxide (CdO) nanoparticles for eliminating cancer cells. J Nanomed Res 2(5): 20.

2. Heidari A, Brown C (2015) Study of surface morphological, phytochemical and structural characteristics of rhodium (III) oxide $\left(\mathrm{Rh}_{2} \mathrm{O}_{3}\right)$ nanoparticles. International Journal of Pharmacology, Phytochemistry and Ethnomedicine 1(1): 15-19.

3. Heidari A (2016) An experimental biospectroscopic study on seminal plasma in determination of semen quality for evaluation of male infertility. Int $J$ Adv Technol 7: e007.

4. Heidari A (2016) Extraction and preconcentration of N-Tolyl-Sulfonyl-PhosphoramidSaeure-Dichlorid as an anti-cancer drug from plants: a pharmacognosy study. $J$ Pharmacogn Nat Prod 2: e103.

5. Heidari A (2016) A thermodynamic study on hydration and dehydration of DNA and RNA-Amphiphile Complexes. J Bioeng Biomed Sci S: 006.

6. Heidari A (2016) Computational studies on molecular structures and carbonyl and ketene groups' effects of singlet and triplet energies of Azidoketene $\mathrm{O}=\mathrm{C}=\mathrm{CH}-\mathrm{NNN}$ and Isocyanatoketene $\mathrm{O}=\mathrm{C}=\mathrm{CH}-\mathrm{N}=\mathrm{C}=\mathrm{O} . J$ Appl Computat Math 5: e142.

7. Heidari A (2016) Study of irradiations to enhance the induces the dissociation of hydrogen bonds between peptide chains and transition from helix structure to random coil structure using ATR-FTIR, Raman and ${ }^{1}$ HNMR Spectroscopies. J Biomol Res Ther 5: e146.

8. Heidari A (2016) Future prospects of point fluorescence spectroscopy, fluorescence imaging and fluorescence endoscopy in photodynamic therapy (PDT) for cancer cells. J Bioanal Biomed 8: e135.

9. Heidari A (2016) A bio-spectroscopic study of DNA density and color role as determining factor for absorbed irradiation in cancer cells. Adv Cancer Prev 1: e102.

10. Heidari A (2016) Manufacturing process of solar cells using cadmium oxide (CdO) and rhodium (III) Oxide $\left(\mathrm{Rh}_{2} \mathrm{O}_{3}\right)$ nanoparticles. J Biotechnol Biomater 6: e125.

Copyright: (C2020 Heidari A. This is an open-access article distributed under the terms of the Creative Commons Attribution License, which permits unrestricted use, distribution, and reproduction in any medium, provided the original author and source are credited. 